\title{
La transmission intergénérationnelle en comportement du consommateur : cadre conceptuel intégrateur et perspectives de recherche
}

\author{
Samuel GUILLEMOT, Univ Brest, LEGO, Quimper, France
}

\begin{abstract}
Résumé : Le concept de transmission intergénérationnelle a donné lieu à des travaux d'une grande hétérogénéité en comportement du consommateur. Il est mobilisé dans des champs de recherche aussi variés que la socialisation du consommateur ou encore la distribution de l'héritage. Cet article propose un cadre conceptuel intégrateur de la transmission intergénérationnelle en comportement du consommateur. Il émerge d'une lecture de la théorie du développement de la personnalité humaine d'Erikson (1959). Le premier apport de ce cadre conceptuel est de relier les préoccupations développementales des jeunes générations à celles des plus anciennes (apprendre, se distinguer, prendre soin et préserver) autour de la notion de capital intergénérationnel. Le second apport est de faire émerger les notions de capital socioculturel, économique, psychique et génétique qui viennent enrichir et préciser la nature du capital intergénérationnel. Enfin, il ouvre des perspectives de recherche telles qu'étudier le rôle central de la génération " pivot » (les 30-65 ans) ou encore prendre en compte le concept d'attachement (Bowbly, 1978) pour étudier l'effet des ressources héritées de nature psychique sur l'adoption de pratiques de consommation.
\end{abstract}

Mots-clés : Héritage - Education - Socialisation - Solidarité - Préservation - Transmission intergénérationnelle - Influences intergénérationnelles - Legs - Don - Echange - Apprentissage

TO CITE: GUILLEMOT S. (2018), LA TRANSMISSION INTERGENERATIONNELLE EN COMPORTEMENT DU CONSOMMATEUR : CADRE CONCEPTUEL INTEGRATEUR ET PERSPECTIVES DE RECHERCHE, RECHERCHE ET APPLICATIONS EN MARKETING, 33 (2), 99-121

Dans nos sociétés contemporaines, des tendances de fonds bouleversent l'articulation traditionnelle des générations et les phénomènes de transmission qui y sont associés. Ces mutations sont tout d'abord d'ordre démographique, avec une durée de vie qui ne cesse de s'allonger entraînant la coexistence de quatre voire cinq générations familiales (Préel, 2005). Par ailleurs, la place et le rôle de chaque génération sont aussi mouvants sous l'effet de mutations sociales : la valorisation de l'individu, l'allongement des périodes de l'adolescence et de la vieillesse (Attias-Donfut et Segalen, 1998), ou encore la place devenue centrale des enfants dans des foyers à géométrie variable ${ }^{1}$ (Ayadi et Gollety, 2012). Enfin, les conditions économiques et politiques favorisent la précarité et une plus grande dépendance des générations les unes par rapport aux autres (Tavoillot et al., 2011). Il est primordial de bien comprendre ces phénomènes de transmission intergénérationnelle car ils sont au cœur des comportements humains. En effet, l'ensemble de nos choix et décisions s'appuie sur un socle de ressources et dispositions qui a été façonné par les générations qui nous ont précédé (Trizzulla et al., 2016). De même, ce que nous souhaitons léguer aux générations qui nous suivent oriente nos choix et décisions (Urien et Kilbourne, 2011).

En comportement du consommateur, on distingue deux principaux cadres conceptuels de référence pour étudier la transmission intergénérationnelle. Le plus ancien fait référence à la socialisation du consommateur. Il s'agit du processus par lequel les individus acquièrent les compétences, connaissances et attitudes nécessaires à la réalisation de leur rôle de consommateur sur le marché (Ward, 1974). La transmission intergénérationnelle concerne dès lors la transmission d'objets immatériels par des interactions implicites/explicites au sein des générations familiales. Le focus est mis sur les enfants consommateurs (e.g. Ward, 1974), les adolescents (e.g. Lueg et al., 2006) ou encore les jeunes adultes (e.g. Moore et al., 2002). Le

\footnotetext{
${ }^{1}$ Famille recomposée, monoparentale, homoparentale, etc.
} 
second cadre s'est développé, depuis les années 2000, sous la pression du vieillissement démographique. Les chercheurs ont commencé à utiliser les théories sur le vieillissement psychologique et la préparation à la mort pour étudier la transmission d'objets matériels, notamment par l'héritage sous la forme de transactions uniques ou répétées (Price et al., 2000 ; Urien, 2003).

Les recherches sur la transmission intergénérationnelle couvrent des objectifs variés (Bourcier-Béquaert et de Barnier, 2010). Certains travaux ambitionnent de favoriser l'éducation des futurs citoyens-consommateurs (Hémar-Nicolas et Rodhain, 2012) ou de comprendre comment les mutations des liens intergénérationnels influent sur les pratiques de consommation (Karanika et Hogg, 2016). D'autres ont une vocation plus managériale : il s'agit de permettre aux marques de s'ancrer dans l'histoire individuelle et familiale (Decoopman et Gentina, 2013 ; Kessous et al., 2015 ; Ladwein et al., 2009) ou encore aux entreprises de concevoir et d'adapter des produits et services répondant aux besoins de transmettre un héritage (Guillemot, 2015 ; Malas, 2012 ; Urien et Guiot, 2007). La variété de ces recherches s'explique par une vision large de la transmission intergénérationnelle. Le concept de «transmission intergénérationnelle » évoque les idées de trajet, de traversée et de passage entre les générations (Gire, 2003). Il est conçu «comme un processus qui met en scène un destinateur [i.e. l'émetteur comme un parent par exemple] et un destinataire [i.e. le receveur comme un enfant par exemple] reliés par une procédure d'interaction » (Lahaye et al., 2007 :109). Ainsi la transmission intergénérationnelle s'entend sous différentes acceptions en comportement du consommateur (tableau 1), selon la nature des objets transmis (immatérielle ou matérielle), selon les processus (interactions implicites/explicites ou transactions uniques/répétées) ou encore selon les acteurs impliqués (génération familiale ou démographique).

Face à cet usage fragmenté du concept de transmission intergénérationnelle en comportement du consommateur, cet article propose un cadre conceptuel intégrateur. $\mathrm{Ce}$ cadre intégrateur est nécessaire afin de livrer une lecture plus complète des transmissions entre générations. En effet la transmission intergénérationnelle est un phénomène dynamique constitué d'influences réciproques entre les générations. Par exemple, les relations grandsparents/petits-enfants ne se limitent pas seulement à des questions d'héritage mais obéissent également à des logiques de socialisation (Derbaix et al., 2015 ; Josion-Portail, 2014). De même, en interagissant avec leurs pairs, les enfants peuvent importer et expérimenter de nouvelles normes dans leurs foyers et ainsi enrichir les pratiques de consommation de leurs parents (Gollety, 1999 ; Gollety et al., 2012).

La construction de ce cadre conceptuel intégrateur se présente de la manière suivante. Dans un premier temps, la théorie du développement de la personnalité humaine d'Erikson (1959) est mobilisée comme grille de lecture pour appréhender la transmission intergénérationnelle. Cette lecture «eriksonienne » permet de mettre en valeur les différentes positions qu'un individu peut occuper vis-à-vis de son capital intergénérationnel : apprendre des autres générations, se distinguer des autres générations, prendre soin (care) des autres générations et préserver pour les autres générations. Le capital intergénérationnel est entendu ici comme un socle d'actifs matériels et immatériels qui se transmet et évolue de génération en génération (Trizzulla et al., 2016). Dans un second temps, les connaissances en comportement du consommateur sont articulées autour de ces quatre positons. C'est à partir de cette lecture que vont émerger les notions de capital socioculturel, économique, psychique et génétique qui vont venir enrichir et préciser la nature du capital intergénérationnel. Le cadre conceptuel intégrateur de la transmission intergénérationnelle prend forme dès lors qu'on articule les différentes composantes du capital intergénérationnel (socioculturelle, économique, psychique et génétique) autour des préoccupations développementales des destinataires et des 
destinateurs (apprendre, se distinguer, prendre soin et préserver). L'article s'achève sur une discussion des apports et voies de recherche ouvertes par la présentation de ce cadre conceptuel intégrateur.

Tableau 1: Les différentes acceptions de la transmission intergénérationnelle en comportement du consommateur

\begin{tabular}{|c|c|c|c|}
\hline & Approches & Descriptions & Illustrations en comportement du consommateur \\
\hline \multirow[t]{2}{*}{$\begin{array}{l}\text { La nature } \\
\text { des objets } \\
\text { transmis } \\
\text { (quoi ?) }\end{array}$} & $\begin{array}{l}\text { Objets } \\
\text { immatériels }\end{array}$ & $\begin{array}{l}\text { Attitudes, préférences, } \\
\text { connaissances, } \\
\text { compétences, pratiques de } \\
\text { consommation, etc. }\end{array}$ & $\begin{array}{l}\text { Le passage à travers les générations des } \\
\text { attitudes (e.g. le scepticisme à l'égard des } \\
\text { publicités, Obermiller et Spangenberg, 2000), } \\
\text { des préférences (e.g. les marques préférées, } \\
\text { Moore et Lutz, 1988), des «savoir-faire » en } \\
\text { matière de consommation (Nancarrow et al., } \\
\text { 2008) ou des pratiques de consommation } \\
\text { (Moore et al., 2002) }\end{array}$ \\
\hline & Objets matériels & $\begin{array}{l}\text { Objets de valeurs, objets } \\
\text { sans valeur financière mais } \\
\text { avec une valeur } \\
\text { sentimentale, argent, etc. }\end{array}$ & $\begin{array}{l}\text { La distribution des objets en fin de vie en guise } \\
\text { d'héritage (Price et al., 2000) }\end{array}$ \\
\hline \multirow{4}{*}{$\begin{array}{l}\text { Les } \\
\text { processus } \\
\text { (Quand et } \\
\text { comment?) }\end{array}$} & $\begin{array}{l}\text { Interactions } \\
\text { explicites }\end{array}$ & $\begin{array}{l}\text { Apprentissage, initiation, } \\
\text { etc. }\end{array}$ & $\begin{array}{l}\text { Les pratiques éducatives des parents relatives à } \\
\text { la consommation (Ayadi et Gollety, 2012); la } \\
\text { transmission d'un savoir-faire et de } \\
\text { connaissances à travers un témoignage } \\
\text { (Guillemot, 2015) }\end{array}$ \\
\hline & $\begin{array}{l}\text { Interactions } \\
\text { implicites }\end{array}$ & $\begin{array}{l}\text { Influences par } \\
\text { imprégnation et/ou par un } \\
\text { processus } \\
\text { d'observation/imitation }\end{array}$ & $\begin{array}{l}\text { Les marques visibles et/ou celles consommées } \\
\text { dans un contexte routinier et fréquent sont } \\
\text { souvent consommées par les enfants lorsqu'ils } \\
\text { quittent le foyer familial (Schindler et al., 2014) }\end{array}$ \\
\hline & $\begin{array}{l}\text { Transactions } \\
\text { répétées }\end{array}$ & Echanges, partages, etc. & $\begin{array}{l}\text { Les échanges et la consommation partagée de } \\
\text { vêtements entre mères et filles (Decoopman et } \\
\text { Gentina, 2013). }\end{array}$ \\
\hline & $\begin{array}{l}\text { Transactions } \\
\text { uniques }\end{array}$ & Dons, legs, etc. & $\begin{array}{l}\text { Le don d'une montre de luxe d'un père à son } \\
\text { fils (Kessous et al., 2015) ; les legs et transferts } \\
\text { financiers (Krebs et al., 2012; Malas et Guiot, } \\
\text { 2010) }\end{array}$ \\
\hline \multirow{2}{*}{$\begin{array}{l}\text { Les } \\
\text { générations } \\
\text { (Qui ?) }\end{array}$} & $\begin{array}{l}\text { Générations } \\
\text { familiales }\end{array}$ & $\begin{array}{l}\text { Succession des } \\
\text { générations en fonction } \\
\text { des liens filiaux : enfants / } \\
\text { parents / grands-parents }\end{array}$ & $\begin{array}{l}\text { Le rôle des enfants (Gollety, 1999), des parents } \\
\text { (Beatty et Talpade, 1994) et/ou des grands- } \\
\text { parents (Derbaix et al., 2015; Josion-Portail, } \\
\text { 2014) dans la construction des } \\
\text { pratiques/connaissances relatives à la } \\
\text { consommation }\end{array}$ \\
\hline & $\begin{array}{l}\text { Générations } \\
\text { démographiques }\end{array}$ & $\begin{array}{l}\text { Succession des } \\
\text { générations en fonction de } \\
\text { l'âge et du cycle de vie : } \\
\text { jeunes / actifs / retraités ou } \\
\text { générations passées / } \\
\text { générations futures }\end{array}$ & $\begin{array}{l}\text { Les pratiques de consommation comme acte de } \\
\text { préservation des conditions de vie des } \\
\text { générations à venir (Lacroix et Jolibert, } 2015 \text {; } \\
\text { Urien et Kilbourne, 2011) }\end{array}$ \\
\hline
\end{tabular}

\section{LA TRANSMISSION INTERGENERATIONNELLE : UNE LECTURE ERIKSONIENNE}

Erik Erikson envisage le développement psychosocial de l'individu comme un processus continu tout au long de la vie. Ces travaux $(1950,1959)$ offrent alors une grille de lecture intéressante pour organiser les recherches en comportement du consommateur allant de la 
socialisation des enfants jusqu'aux pratiques de distribution de l'héritage des personnes âgées. Erikson est un psychanalyste américain né au début du $20^{\text {ème }}$ siècle spécialisé dans le développement psychosocial des enfants. Il a notamment travaillé dans les années 1930 dans les réserves indiennes sioux et yurok aux Etats-Unis d'Amériques. Erikson se rend compte que l'origine de certains problèmes psychosociaux des Indiens est à chercher dans le décalage entre l'histoire et les traditions de leurs tribus, et leurs modes de vie dans les réserves. C'est sur la base de ses observations comparées et transposées à la culture occidentale qu'il forme sa théorie du développement de la personnalité. Il souligne les aspects psychosociaux, culturels et historiques du développement de l'ego. Il insiste sur le fait que la personnalité se développe dans une réalité sociale, au sein de groupe(s) avec le(s)quels nous avons un passé commun mais également un futur partagé.

Le passé commun fait référence aux relations familiales et sociales, et aux traditions culturelles qui façonnent les comportements. Il propose un modèle du développement des enfants et adolescents par étapes qu'il met en parallèle avec les thèses freudiennes du développement par stades (oral, anal, etc.). Ces étapes prennent également en compte les interactions avec l'environnement qui se complexifient à mesure que l'enfant grandit (la mère, les parents, la famille, l'école puis les amis, etc.). Plus précisément Erikson (1950) propose initialement une succession de six étapes durant lesquelles l'enfant puis l'adolescent doit trouver un équilibre entre deux pôles opposés. Par exemple lors de la première phase du développement (0-18 mois), le nouveau-né doit trouver un équilibre entre un attachement inconditionnel à sa mère (la confiance) et une protection contre le monde extérieur (la méfiance). La démarche proposée ici ne consiste pas à aborder le développement étape par étape, mais plutôt de s'en inspirer en restant au niveau plus général de la relation entre générations. En ce sens nous nous intéressons à la position vis-à-vis d'un capital intergénérationnel - un socle d'actifs matériels et immatériels- qui se transmet et évolue de génération en génération. L'enfant va d'abord s'imprégner de ce capital, puis il va, en grandissant y puiser des ressources et des dispositions qu'il va articuler en fonction de ses préoccupations identitaires (Trizzulla et al., 2016). C'est d'ailleurs, en partie, la démarche proposée par Ladwein et al. (2009) ${ }^{2}$ dans une recherche portant sur la socialisation à la consommation des jeunes-filles par leurs mères. Les auteurs y décrivent comment se construit dès l'enfance un socle d'habitudes et de connaissances (concernant notamment les pratiques et usages, le choix des marques, des produits et des circuits de distribution) qui va servir de référentiel disponible en situation d'achat ou de consommation tout au long de la vie.

L'intérêt de la démarche d'Erikson (1959) est d'associer à ce passé commun, la vision d'un futur partagé. Profondément marqué par le second conflit mondial - Erikson, né allemand, a vu sa famille d'origine Juive émigrer aux Etats-Unis sous la pression du régime Nazi- il est convaincu que l'éducation et la transmission des valeurs collectivement partagées sont essentielles au bon développement des individus et des sociétés. Il enrichit donc sa théorie de deux étapes supplémentaires durant lesquelles le fait de prendre soin (care) du capital intergénérationnel et d'en préserver les aspects jugés essentiels deviennent des préoccupations $\mathrm{du}$ développement psychosocial des adultes. La notion de transmission devient alors essentielle dans sa théorie puisqu'elle articule les préoccupations développementales des jeunes générations avec celles des plus anciennes.

En synthèse, une lecture eriksionnenne de la transmission intergénérationnelle permet d'identifier quatre positions essentielles vis-à-vis du capital intergénérationnel pendant lesquelles les générations ont besoin les unes des autres pour se développer : L’individu doit

\footnotetext{
${ }^{2}$ Ladwein et al. (2009) introduisent la notion de «capital transgénérationnel» car ils se focalisent exclusivement sur le point de vue des destinataires (les jeunes filles). Dans cet article, le préfixe «inter» est privilégié (capital intergénérationnel) car les points de vue des destinataires et des destinateurs sont considérés.
} 
d'abord apprendre des générations qui le précèdent les compétences et les méthodes nécessaires à la vie en société. Il doit ensuite s'en distinguer en développant ses propres identités et buts dans la vie. Il est alors prêt à prendre soin (care) des générations qui le suivent, puis à préserver des éléments qui pourront leur être utiles. Le tableau 2 présente ces préoccupations et $\mathrm{y}$ associe les recherches en comportement du consommateur et les principales problématiques développées dans ces travaux.

Près d'une centaine de travaux en comportement du consommateur ont été mobilisés pour réaliser cet état des connaissances. Ils ont été identifiés de la manière suivante. Une première approche a consisté à consulter les bases de données de 45 des plus importantes revues marketing francophones et internationales ${ }^{3}$ avec comme mot-clé «intergénérationnel » et «transmission intergénérationnelle » en français et en anglais. 39 articles relevant explicitement des transmissions intergénérationnelles ont ainsi pu être identifiés sur la période 1970-2017. Dans un second temps, les bibliographies de ces articles ont été examinées et les travaux clés sur lesquels s'étaient appuyés ces articles ont été intégrés à notre synthèse. Cela a permis de dégager les deux cadres d'analyse présentés en introduction : la socialisation des enfants à la consommation par la transmission d'actifs immatériels (avec Ward (1974) comme article fondateur) et la distribution de l'héritage des personnes âgées par la transmission d'actifs matériels (avec Price et al. (2000) pour article fondateur). A partir de là, la recherche de références bibliographiques a été diversifiée à l'aide de nouveaux mots clés tels « socialisation », « influences » et « apprentissages » pour identifier des travaux relevant de la transmission d'actifs immatériels; et « héritage », « échanges », « dons » et «legs » pour la transmission d'actifs matériels. Ont été retenus les travaux (articles, livres, thèses, actes de conférences) qui examinent les relations impliquant au moins deux membres de générations familiales et/ou démographiques - distinctes.

La synthèse des connaissances issues de ces travaux est présentée dans les quatre parties suivantes qui correspondent aux quatre positions vis-à-vis du capital intergénérationnel (tableau 2).

\footnotetext{
${ }^{3}$ Ces revues sont celles identifiées par la FNEGE (Fondation Nationale pour l'Enseignement de la Gestion des Entreprises) comme revues scientifiques de référence. La FNEGE s'appuie sur un collège scientifique regroupant l'ensemble des associations savantes françaises en sciences de gestion pour établir un classement des revues scientifiques actualisé tous les trois ans. Ces 45 revues scientifiques de référence dans le domaine du marketing s'appuient toutes sur un comité de lecture composé de chercheurs reconnus avec une procédure d'évaluation rigoureuse en double aveugle. Les revues sont classées selon un ensemble de critères objectifs tels que leur présence dans les classements établis par des organismes d'évaluation étrangers et français (comme le CNRS), et par un ensemble d'indices bibliographiques (scores d'impact avec et sans autocitations, indice h, indice d'impact SJR).
} 
Tableau 2 : Préoccupations développementales et positions vis-à-vis du capital intergénérationnel (adapté d'Erikson, 1959)

\begin{tabular}{|c|c|c|c|c|}
\hline $\begin{array}{l}\text { Préoccupations } \\
\text { développementales }\end{array}$ & Temps de la vie & Relations privilégiées & $\begin{array}{l}\text { Recherches en } \\
\text { comportement du } \\
\text { consommateur }\end{array}$ & Principales problématiques développées \\
\hline $\begin{array}{l}\text { Apprendre des autres } \\
\text { générations } \\
\text { Apprendre des } \\
\text { compétences et des } \\
\text { méthodes; Assimiler les } \\
\text { valeurs et les } \\
\text { comportements valorisés }\end{array}$ & $\begin{array}{l}\text { Principalement le } \\
\text { temps de l'enfance }\end{array}$ & $\begin{array}{l}\text { Parents, le reste de la } \\
\text { famille puis } \\
\text { progressivement les } \\
\text { amis et autres } \\
\text { référents (école, etc.) }\end{array}$ & $\begin{array}{l}\text { Les pratiques } \\
\text { éducatives et les } \\
\text { influences } \\
\text { intergénérationnelles } \\
\text { au sein du foyer }\end{array}$ & $\begin{array}{l}\text { Identifier les contenus des apprentissages familiaux (Gollety et al., } \\
2012 \text {; Moore et al., } 2002 \text {; Schindler et al., } 2014 \text {; Ward, 1974) } \\
\text { Comprendre les processus de formation des apprentissages familiaux } \\
\text { (Ayadi et Gollety, } 2012 \text {; Carlson et Grossbart, } 1988 \text {; Mandrik et al., } \\
\text { 2005) } \\
\text { Comprendre les rôles et apports des différents membres de la famille } \\
\text { (Beatty et Talpade, } 1994 \text {; Derbaix et al., } 2015 \text {; Gollety, } 1999 \text {; Josion- } \\
\text { Portail, 2014) }\end{array}$ \\
\hline $\begin{array}{l}\text { Se distinguer des autres } \\
\text { générations } \\
\text { Définir et développer ses } \\
\text { identités personnelles et } \\
\text { sociales ; Définir des buts } \\
\text { et choisir des directions } \\
\text { dans la vie }\end{array}$ & $\begin{array}{l}\text { Principalement } \\
\text { l'adolescence et le } \\
\text { début de la vie } \\
\text { adulte }\end{array}$ & $\begin{array}{l}\text { Pairs, groupe d'amis, } \\
\text { personnes ayant des } \\
\text { affinités identitaires }\end{array}$ & $\begin{array}{l}\text { L'impact et la } \\
\text { contribution des } \\
\text { ressources transmises } \\
\text { à la construction des } \\
\text { valeurs et pratiques de } \\
\text { consommation }\end{array}$ & $\begin{array}{l}\text { Comprendre les emprunts et les rejets des pratiques de consommation } \\
\text { entre générations (Decoopman et Gentina, 2013; Ladwein et al., 2009) } \\
\text { Préciser le rôle des ressources disponibles de nature : } \\
\text { socioculturelles (Ladwein et al., 2009; Moore et al., 2002) } \\
\quad \text { psychiques (Chaplin et Roedder-John, } 2010 \text {; Richin et } \\
\text { Chaplin, 2015 ; Rindfleisch et al., 1997) } \\
\quad \text { génétiques (Simonson et Sella, 2011) }\end{array}$ \\
\hline $\begin{array}{l}\text { Prendre soin des autres } \\
\text { générations } \\
\text { Guider et prendre soin } \\
\text { (care) des plus jeunes et/ou } \\
\text { des plus âgés }\end{array}$ & $\begin{array}{l}\text { Principalement la } \\
\text { vie adulte (la mi- } \\
\text { vie) }\end{array}$ & $\begin{array}{l}\text { Les enfants, la } \\
\text { famille, sa } \\
\text { communauté } \\
\text { d'appartenance }\end{array}$ & $\begin{array}{l}\text { Les pratiques de } \\
\text { solidarités entre } \\
\text { générations }\end{array}$ & $\begin{array}{l}\text { Comprendre les mécanismes et motivations liés aux transferts } \\
\text { économiques et matériels dans les moments de crise (Karanika et Hogg, } \\
\text { 2016, Noble et al., 2004) } \\
\text { Décrire les caractéristiques et le rôle des aidants familiaux (Mahur et } \\
\text { Moschis, 1999) }\end{array}$ \\
\hline $\begin{array}{l}\text { Préserver pour les autres } \\
\text { générations } \\
\text { Préserver de la mort et de } \\
\text { l'oubli ce qui compte } \\
\text { vraiment, ce qui a de la } \\
\text { valeur (héritage) }\end{array}$ & $\begin{array}{l}\text { Principalement la } \\
\text { dernière partie de } \\
\text { la vie }\end{array}$ & $\begin{array}{l}\text { Famille, personnes } \\
\text { ayant des affinités } \\
\text { identitaires, la } \\
\text { société, le monde }\end{array}$ & $\begin{array}{l}\text { Les pratiques de } \\
\text { construction et de } \\
\text { transmission de } \\
\text { l'héritage }\end{array}$ & $\begin{array}{l}\text { Comprendre les motivations et les choix des destinataires par les destinateurs } \\
\text { (Price et al., } 2000 \text {; Guillemot } 2015 \text {; Malas et Guiot, 2010) } \\
\text { Identifier les supports de significations des héritages (Guillemot et Urien, 2010, } \\
\text { Price et al., } 2000 \text {; Rieunier et Urien, 2011) } \\
\text { Comprendre la perception et l'utilisation de l'héritage comme ressources par les } \\
\text { destinataires (Curasi et al., } 2004 \text {; Kessous et al., 2015) } \\
\text { Décrire le rôle de gardien de l'histoire familiale (Curasi et al., 2004) et/ou de } \\
\text { passeur des significations collectives (Guillemot et Urien, 2016) }\end{array}$ \\
\hline
\end{tabular}




\section{APPRENDRE DES AUTRES GENERATIONS}

La première phase du développement de la personnalité consiste à s'imprégner de son capital intergénérationnel. Il s'agit de se construire par rapport aux autres générations (première ligne du tableau 2). Dans la théorie originelle d'Erikson, il s'agit d'une phase essentielle du développement, constituée de plusieurs étapes en fonction du développement cognitif, moteur et social (Erikson, 1950). Elle concerne principalement le temps de l'enfance. L'enfant a besoin des générations qui le précèdent pour décrypter et comprendre les codes de la vie en société, acquérir des méthodes et des compétences. D'abord passif et dépendant de ses parents, l'enfant va progressivement élargir son horizon à d'autres sources de référence : le reste de sa famille, l'école, les médias, etc. En comportement du consommateur, les travaux qui traitent du sujet s'inscrivent dans le champ d'étude relatif à la socialisation des consommateurs (Ward, 1974). La transmission intergénérationnelle - entendue ici sous l'acception du passage d'objets immatériels au sein des générations familiales - est l'un des moyens de socialisation le plus important. Les enfants ont en effet beaucoup de temps pour apprendre et intégrer comme normes, les attitudes et comportements de leurs parents (e.g. Moschis et Churchill, 1978; Moschis et Moore, 1979; Ward et al., 1977). Depuis une quarantaine d'années, les chercheurs poursuivent l'objectif de comprendre le rôle de la famille dans l'apprentissage social de la consommation et plus particulièrement tentent de répondre à deux questions : qu'est-ce qui se transmet ? et comment ? (Moore et al., 2002 ; Ward, 1974). Ces travaux mettent en évidence l'existence d'un socle d'actifs intangibles relatif au " savoirconsommer » qui se transmet de génération en génération et que l'on appellera ici le « capital socioculturel ».

La première section ci-dessous présente ce capital socioculturel et précise son contenu. La seconde section se focalise sur les types d'influences à l'origine de sa transmission d'une génération à une autre.

\section{La notion de capital socioculturel}

Dès les années 1970, les chercheurs se rendent compte qu'il existe des continuités entre les comportements de consommation des parents et ceux de leurs enfants. La notion de capital socioculturel est évoquée lorsque ces continuités sont envisagées comme un socle de compétences, connaissances et d'attitudes relatif à la consommation qui se construit dès l'enfance (Ladwein et al., 2009). Cette vision prolonge la théorie sociologique de la transmission socioculturelle de l'agent (Bourdieu, 1994 ; Lahaye et al., 2007) dans le champ de la consommation. Celle-ci postule que les normes, pratiques, habitudes et symboles utiles au vivre en société se transmettent de génération en génération à travers des groupes sociaux institués comme la famille, l'école, etc. (Bourdieu, 1979). De nombreuses recherches empiriques ont été menées dans le but d'identifier et de mesurer les continuités dans la consommation engendrée par la filiation. Ces travaux mettent en œuvre des méthodologies basées principalement sur l'autoévaluation subjective des acteurs ou sur l'évaluation croisée de dyade destinateur/destinataire (tableau 3). Ils ont permis d'identifier des continuités intergénérationnelles en ce qui concerne les connaissances (e.g. ensemble évoqué de marques, e.g. Moore et al., 2002), les «savoir-faire» (e.g. Mandrick, Fern et Bao, 2005 ; Nancarrow, Tinson et Brace, 2008) et pratiques de consommation (e.g. de la stratégie de planification des courses à la manière de ranger et consommer ses achats, e.g. Moore-Shay et Lutz, 1988). La reproduction intergénérationnelle concerne également certaines attitudes comme les préférences (e.g. Derbaix et al., 2016 ; Moore et al., 2002 ; Siddiqui et al., 2012), la fidélité aux marques (e.g. Cai, Zhao et He, 2015), la sensibilité aux promotions (e.g. Schindler, Lala et Corcan, 2014), la sensibilité à l'innovation (Cotte et Wood, 2004), ou encore le scepticisme 
au regard des publicités (e.g. Obermiller et Spangenberg, 2000), etc. D’une manière générale, la consommation peut être considérée comme un vecteur pour faire passer des valeurs d'une génération à une autre (e.g. le matérialisme, Moschis et Churchill, 1978 ; Moschis et Moore, 1979).

Tableau 3: Principales méthodologies déployées pour identifier les continuités et les évolutions entre générations

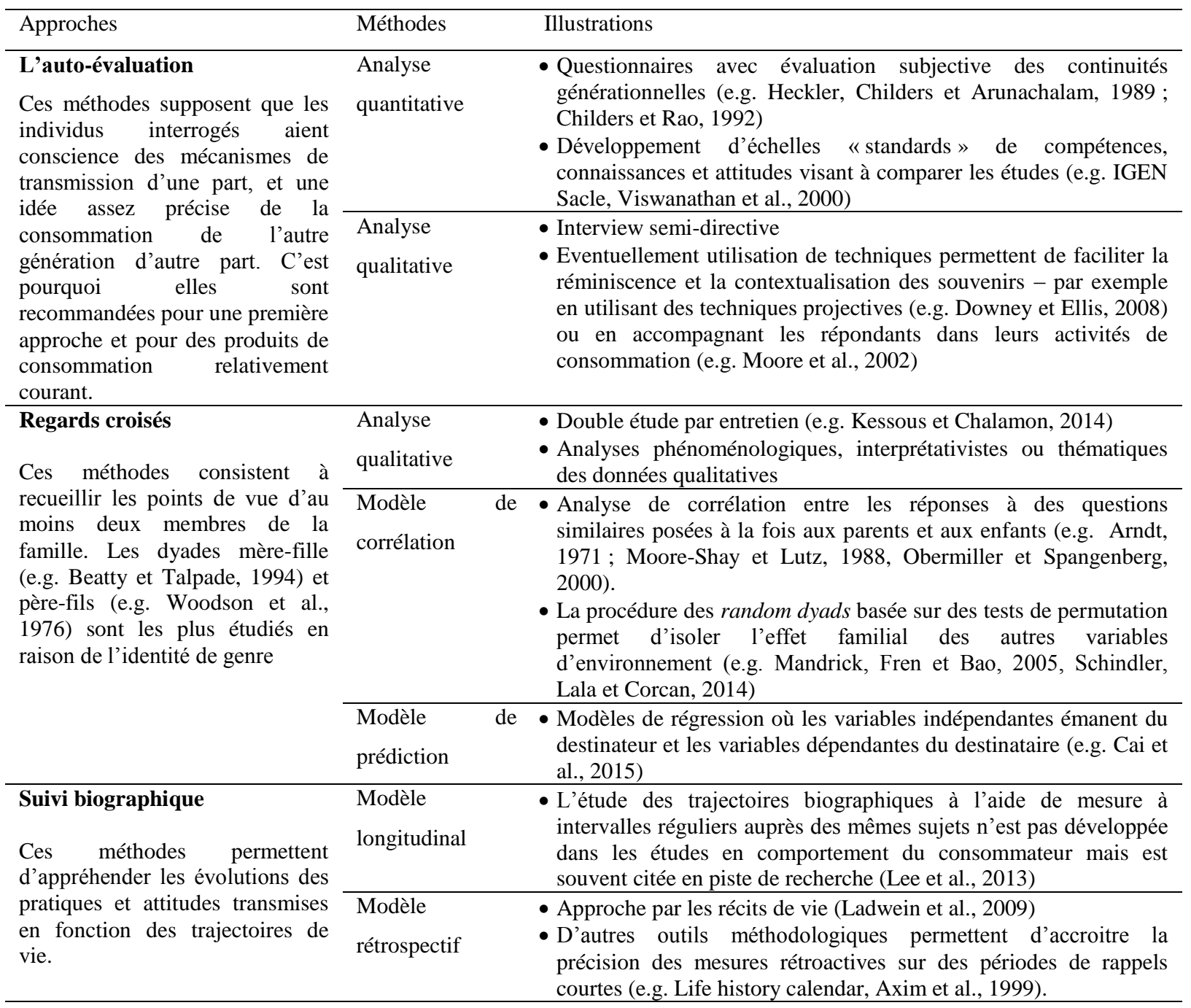

\section{Les types d'influences à l'origine de la transmission du capital socioculturel}

Deux types d'influences permettent d'expliquer la transmission du capital socioculturel entre les générations : les influences implicites à la base de l'apprentissage par observation/imitation, d'une part et les influences explicites incarnées dans les pratiques éducatives et les styles parentaux, d'autre part. Qu'elles soient implicites ou explicites, les chercheurs soulignent le caractère réciproque de ces influences intergénérationnelles.

Les influences implicites. Les compétences, connaissances et attitudes relatives à la consommation s'acquièrent progressivement en grande partie de manière implicite par un mécanisme d'observation/imitation (Ayadi et Gollety, 2012). Tout au long de leur développement, les enfants ont en effet de nombreuses opportunités pour observer les 
attitudes, les préférences et les comportements des adultes et de les intégrer comme des normes (Moschis et Churchill, 1978 ; Moschis et al., 1984). Ainsi, la probabilité que les enfants adoptent les préférences de leurs parents est plus forte pour la consommation de produits et de marques « visibles » dans le foyer (Moore et al., 2002), pour les comportements routiniers et fréquents (Schindler et al., 2014), ou encore pour les attitudes concrètes plutôt qu'abstraites (Moschis et Churchill, 1978 ; Mandrik et al., 2005). Les recherches s'attachent à contextualiser la place de ces apprentissages familiaux au regard du développement des capacités cognitives de l'enfant (Roedder-John, 1999) et des autres sources de socialisation comme l'influence des pairs, des médias et de l'école. Ainsi lorsque l'enfant grandit, l'influence de la famille reste prépondérante pour les produits et consommations réalisées dans le cadre privé du foyer (Childers et Rao, 1992) mais diminue au profit de celle des pairs pour les produits et consommations à caractère social et public (Beatty et Talpade, 1994).

Les influences explicites. Si l'éducation parentale à la consommation opère en grande partie de manière implicite par l'observation et l'imitation, le passage à l'explicite engage les parents à réfléchir aux contenus de leurs apprentissages et à faire attention à la cohérence entre les discours et les comportements réels. A ce titre, Ayadi et Gollety (2012) identifient plusieurs rôles joués par les parents lors de l'apprentissage du savoir consommer. Le rôle d'éducateur consiste à expliquer formellement les choix et décisions et à motiver les refus. Le rôle de stimulateur de consommation permet de donner aux enfants de l'autonomie en les faisant participer aux décisions de la famille. Enfin, le rôle de médiateur permet de mettre en perspective et de faciliter la compréhension des informations provenant des autres sources de socialisation telles les médias et les pairs. En ce sens, les chercheurs se sont intéressés à la qualité de la communication au sein du foyer (Cai et al., 2015 ; Moschis et Moore, 1979 ; Schindler et al., 2014 ; Viswanathan et al., 2000) et à l'impact des différents styles parentaux sur l'apprentissage social de la consommation (Beatty et Talpade, 1994; Carlson et Grossbart, 1988 ; Heckler et al., 1989, Wiman, 1983). En synthèse, les parents qui cherchent à responsabiliser leurs enfants ont plutôt tendance à expliquer leurs choix, ce qui facilite l'intégration et la compréhension des règles du savoir consommer (Carlson et al., 1994). Au contraire, les parents qui recherchent l'harmonie et/ou qui n'expliquent pas, ou peu, leurs choix sont susceptibles d'engendrer de la confusion chez les enfants (Kim et al., 2009).

Des influences implicites et explicites réciproques. Les chercheurs ont mis en évidence le caractère dynamique de l'apprentissage social de la consommation par des échanges et influences (implicites ou explicites) réciproques. Le phénomène de «socialisation inversée » décrit comment les enfants enrichissent les pratiques de consommation de la famille : en interagissant avec d'autres sphères sociales que celle de leurs parents, les enfants expérimentent de nouvelles normes, de nouvelles pratiques qu'ils peuvent introduire dans leur foyer (Gollety, 1999; Gollety et al., 2012). L'avis des enfants sera particulièrement suivi concernant les produits et/ou comportements liés aux nouvelles technologies (Shah et Mittal, 1997), ou aux évolutions des modes de vie (Lueg et al., 2006). D'autre part, le modèle traditionnel de la famille évoluant, l'étude des influences au sein des dyades de genre père-fils (e.g. Woodson et al., 1976) ou mère-fille (e.g. Beatty et Talpade, 1994) s'est progressivement enrichit en intégrant l'influence d'autres membres de la famille (Cotte et Wood, 2004) comme les frères et sœurs et les grands-parents. Ces derniers participent à la socialisation de leurs petits-enfants tout en étant généralement plus «souples » que les parents (Josion-Portail et Michel, 2016). En matière de contenu, ils contribuent à l'émergence de connaissances concernant la nature, la culture, les activités sportives, mais aussi au développement du goût et de la préférence pour certains produits/activités (Derbaix et al., 2015). 


\section{SE DISTINGUER DES AUTRES GENERATIONS}

Une fois les normes et références du capital intergénérationnel assimilés, l'individu va petit à petit chercher à se singulariser et à se différencier des générations qui le précèdent (seconde ligne du tableau 2). Cette tâche développementale est difficile et les individus s'appuient sur les ressources déjà disponibles. En comportement du consommateur, les chercheurs s'intéressent aux ressources transmises - par les parents notamment - et à leurs contributions à la formation des valeurs de consommation et aux choix des pratiques de consommation de leurs enfants.

La première section s'intéresse aux ressources de nature socioculturelle. Les travaux présentés s'ancrent toujours dans le courant de la socialisation du consommateur. Mais le focus est davantage mis sur la génération qui reçoit (Ladwein et al., 2009 ; Moore et al., 2002). La problématique consiste à déterminer pourquoi le destinataire va mobiliser, rejeter, ou faire évoluer certaines compétences, connaissances et attitudes issues de son capital socioculturel hérité.

Les deux sections suivantes mettent en valeur les effets respectifs des ressources héritées de nature psychique et génétique sur les choix et décisions de consommation. Les travaux présentés permettent d'introduire les composantes psychique et génétique du capital intergénérationnel.

\section{L'individualisation du capital socioculturel}

Le capital socioculturel sert de référentiel disponible en situation d'achat ou de consommation (Ladwein et al., 2009). Les individus vont néanmoins adapter et réinterpréter ce qui leur a été transmis en fonction de leur environnement, de leurs choix identitaires et de la direction qu'ils souhaitent prendre dans la vie (Trizzulla et al., 2016). Par exemple, la fille qui quitte le foyer familial aura tendance à rejeter certaines pratiques de sa mère, particulièrement celles associées au rôle domestique, pour marquer sa singularité. Néanmoins, ces rejets sont souvent partiels et circonstanciés : lorsque la fille devient mère à son tour, elle aura tendance à réinvestir les pratiques transmises (Ladwein et al., 2009). Ces comportements s'expliquent par la théorie sociologique de la transmission socioculturelle de l'acteur (Lahaye et al., 2007). Cette théorie postule que l'individu a la capacité de prendre conscience de l'influence qu'exerce son passé sur sa propre personne (Attias-Donfut, 2000). L'individualisation du capital socioculturel est alors une notion essentielle : elle permet à l'individu de garder son identité, son histoire tout en intégrant les changements qui se produisent autour de lui (Trizzulla et al., 2016). Il convient de noter encore une fois les caractères réciproque et dynamique de ces influences intergénérationnelles. En montrant que certaines mères peuvent modifier leurs préférences pour des marques et/ou des enseignes vestimentaires dans des logiques de comparaison sociale et symbolique avec leur fille, Deccopman et Gentina (2013) montrent bien que le capital socioculturel des parents évolue sous l'influence de leurs enfants.

\section{La notion de capital psychique}

Si le cycle des générations est traversé par des considérations socioculturelles, celles-ci engendrent des relations entre les personnes. Le lien social et l'affect deviennent alors des 
vecteurs importants de la transmission. A ce stade, nous introduisons la notion de «capital psychique » qui constitue un prolongement des théories psychiques sur l'attachement (Bowbly, 1978). Les recherches en psychologie et en psychologie sociale s'appuient sur le fait que l'attention et le support parental reçu pendant l'enfance impactent durablement le développement psychologique de l'individu à travers son niveau d'estime de soi et d'in/sécurité perçue (Bowbly, 1978). Ces styles psychiques ont tendance à se répéter entrainant les générations dans des cercles vertueux et/ou vicieux dont il est difficile de s'extraire car l'individu n'a pas forcément conscience du phénomène (Kaës et al., 2003 ; Winter, 2012). L'individu confronté à un sentiment d'insécurité psychique ou à une faible estime de lui-même va chercher du réconfort en adoptant des comportements qu'il suppose valorisant (Chaplin et Roedder John, 2010). Ainsi, il a été démontré que la perception d'un sentiment d'insécurité psychique - lié à une absence d'attention/support parental pendant l'enfance - peut être considérée comme une variable antécédente de l'adoption de valeurs matérialistes (Felfoul, 2014 ; Richins et Chaplin, 2015 ; Robert et al., 2003 ; Rindfleish et al., 1997 ; Rindfleisch et al., 2009). De la même manière un faible niveau d'estime de soi permet de prédire le niveau de matérialisme des adolescents (Chaplin et Roedder John, 2010) ou encore la tendance des mères à co-consommer et échanger des vêtements avec leur fille adolescente (Gentina et al., 2013). En ce sens, on peut considérer que le capital psychique peut influencer la manière dont un individu va intégrer et s'approprier les compétences, connaissances et attitudes issues de son capital socioculturel.

\section{La notion de capital génétique}

Les travaux présentés précédemment mettent l'accent sur l'impact qu'exercent les enjeux socioculturels, économiques et psychiques sur les générations. Néanmoins, ces influences sont contenues dans un cadre prédéterminé par la biologie. Les études menées en biologie comportementale - l'étude des effets des gènes sur les comportements (Plomin et al., 1999) tendent à démontrer que l'individu structure son environnement en fonction de son capital génétique (Johnson et al., 2009). Le capital génétique fait ainsi référence aux prédispositions génétiques à effectuer/s'engager dans des choix/comportements (Plomin et al., 1999). Bien entendu, les gènes n'influencent pas directement le comportement, ils le font en "codant» des séquences d'acides aminés qui forment des protéines. Ce sont ces protéines qui « créent le système squelettique, les muscles, ainsi que les systèmes endocrinien, immunitaire, digestif, et le système nerveux, ce dernier étant le plus important pour le comportement » (Plomin et al., 1999 :61). Pourtant, le fait que tous les comportements aient une composante héritable ne signifie pas que l'effet des gènes soit toujours important. La variance dans les aptitudes et comportements due à la génétique oscille généralement entre 25 et $30 \%$ et n'excède jamais $50 \%$ (Turkheimer, 2000).

Simonson et Sela (2011) ont cherché à déterminer l'influence réelle des gènes sur les choix et décisions de consommation en confrontant des paires de jumeaux monozygote $(100 \%$ du patrimoine génétique identique, $\mathrm{n}=220$, âge moyen 46,6 ans) et dizygote ( $50 \%$ du patrimoine génétique partagé, $\mathrm{n}=140$, âge moyen 49 ans) à une série de tests et problèmes de décisions (decision making and behavioral decision measures). Les résultats indiquent une héritabilité génétique très faible - souvent insignifiante - sur une grande partie des choix et décisions de consommation, sauf lorsqu'interviennent les notions de prudence et de prise de risque. Ces résultats suggèrent que les effets génétiques seraient liés à des tendances générales de la personnalité (la tendance à la prise de risque ici) qui peuvent se manifester dans les choix identitaires et les prises de décisions de consommation. Néanmoins ces résultats restent 
exploratoires et nécessitent des recherches complémentaires pour préciser le rôle du capital génétique sur les choix et décisions de consommation.

\section{PRENDRE SOIN DES AUTRES GENERATIONS}

La vie adulte est la période où s'articulent et se mêlent les liens du passé, du présent et du futur. C'est un moment charnière où l'individu réalise pleinement l'importance des liens intergénérationnels et la valeur de ses héritages. Erikson parle de générativité pour désigner un instinct de communion qui pousse l'individu à investir une partie de lui-même pour contribuer au bien-être des autres générations (troisième ligne du tableau 2). Dans cette configuration, la transmission intergénérationnelle peut être considérée comme une forme particulière de don où des acteurs de générations différentes sont impliqués. Par conséquent, l'obligation de rendre est différée dans le temps : on prend soin de la génération de ses enfants pour «payer sa dette » vis-à-vis des générations précédentes; et les enfants le rendront en s'occupant de leurs parents lorsqu'ils vieilliront (Attias-Donfut et Segalen, 1998). En comportement du consommateur, hormis les pratiques éducatives précédemment évoquées, la thématique des solidarités intergénérationnelles est peu développée. On ne peut pas encore parler de courant de recherche avec une origine clairement identifiable comme peuvent l'être les travaux de Ward (1974) pour la socialisation du consommateur. On trouve néanmoins dans la littérature quelques travaux qui s'intéressent aux soutiens financiers et matériels apportés par des individus à leurs enfants adultes (e.g. Karanika et Hogg, 2016) et/ ou à leurs parents âgés (e.g. Mahur et Moschis, 1999), et à leurs impacts sur les pratiques de consommation.

La première section introduit ainsi une nouvelle composante du capital intergénérationnel le capital économique - qui se fonde sur la vision des sciences économiques du capital. La seconde section envisage l'impact de ce capital économique sur les comportements de consommation.

\section{La notion de capital économique}

En économie, la notion de capital englobe les actifs matériels et les flux financiers qui se transmettent à travers les générations (Piketty, 2013). Les sciences économiques éclairent les mouvements de reproduction de ce capital à travers le temps et constatent un retour des sociétés dominées par le poids de l'héritage (Arrondel et Masson, 2011). Comme l'explique Piketty $(2013: 601)$ «dans la mesure où le XXI ${ }^{\text {ème }}$ siècle se caractérisera par un abaissement de la croissance (démographique et économique) et un rendement du capital élevé (dans un contexte de concurrence exacerbée entre pays pour attirer les capitaux), ou tout du moins dans les pays où une telle évolution se produira, l'héritage retrouvera donc sans doute une importance voisine de celle qui était la sienne au XIX ${ }^{\mathrm{ème}}$ siècle ». Hormis les problématiques liées spécifiquement à la succession qui seront abordées plus loin dans l'article, de nombreux transferts entre générations ont lieu tout au long de la vie. Ces flux sont difficiles à appréhender car d'une part, ils sont peu visibles et d'autre part, ils relèvent d'une multitude de motivations : aides familiales en guise de solidarité, cadeaux pour faire plaisir ou avantages fiscaux, etc. (Gale et Scholtz, 1994 ; Masson, 2009). Cela peut expliquer le peu de travaux académiques sur le sujet. Pourtant les ressources économiques et les conditions de vie matérielles de la famille influencent la consommation des enfants qui en sont issus. Par exemple, la présence ou non de donations significatives détermine de nombreux choix personnels et familiaux comme le choix du logement (Piketty, 2013). C'est pourquoi dès la fin des années 1970, Moschis et ses collègues considéraient le statut socio-économique de la 
famille pour appréhender la socialisation du consommateur (Moschis et Churchill, 1978 ; Moschis et Moore, 1979).

\section{L'influence du capital économique sur les comportements de consommation}

Par rapport aux sciences économiques qui étudient les mouvements d'ensemble du capital, les travaux en comportement du consommateur apportent une vision centrée sur les individus en explorant les motivations et les significations données par les acteurs à la transmission de ce capital économique. Les quelques travaux qui traitent du sujet dans notre discipline mettent en avant plusieurs formes de solidarités. La solidarité «descendante » fait référence à la situation d'adultes qui reçoivent un soutien matériel et financier de leurs parents (e.g. Karanika et Hogg, 2016). C'est la forme la plus commune sachant que $90 \%$ des flux financiers entre générations sont effectués des plus âgés vers les plus jeunes (Cordier, Houdré et Ruiz, 2007). La solidarité «ascendante» fait référence, quant à elle, aux enfants qui prennent soin de leurs parents âgés (e.g. Mahur et Moschis, 1999). Dans les deux cas, le rôle de personnes centrales dans la famille - les «caregivers »- est mis en avant. Mahur et Moschis (1999) révèlent une forme de déterminisme familial et social dans l'adoption de ce rôle : la personne qui prend soin des autres est le plus souvent prédisposée par son genre (une femme), son rang dans sa fratrie (l'ainé.e) et/ou par la proximité géographique des personnes aidées. Ces situations de solidarité créent des sentiments ambivalents allant de l'amour pour les destinateurs à la honte pour les destinataires (Karanika et Hogg, 2016). Ces sentiments ont un impact sur les comportements de consommation quotidiens dans le sens où les acteurs vont avoir tendance à délaisser une partie de leurs identités personnelles au profit de leurs identités familiales et collectives (Karanika et Hogg, 2016). Ces résultats suggèrent donc que le capital économique distribué à l'occasion des solidarités intergénérationnelles va influencer la manière dont les individus vont individualiser leur capital socioculturel à travers leurs choix et pratiques de consommation. Cela reste un point à approfondir et sera développé dans les perspectives de recherche en fin d'article.

\section{PRESERVER POUR LES AUTRES GENERATIONS}

Lorsque l'idée et la réalité de la mort se font sentir (le temps de la vieillesse, dernière ligne du tableau 2), l'individu craint que tout ce qu'il a connu et construit disparaisse avec lui. Il se livre à un travail de réflexion sous forme de bilans multiples qui lui permet de reconstruire le passé en fonction de l'image qu'il veut laisser après la mort (Butler, 1963). Ces réflexions approfondies vont apporter de nouveaux éclairages sur certains événements de la vie et mettre en valeur des aspects saillants de l'identité individuelle et familiale qu'il va chercher à transmettre pour les préserver de la mort (McAdams, 2001; Vaillant, 2002). En comportement du consommateur, un des premiers articles à s'intéresser au sujet est celui de Price et al. (2000) qui montre comment les personnes âgées préservent des significations personnelles et familiales à travers la distribution de leurs objets. Dès lors, plusieurs travaux ont étudié les pratiques de construction et de distribution de l'héritage. Ils ont confirmé le rôle prépondérant des significations associées à l'héritage (Guillemot 2015). La première section présente le cas où ces significations s'incarnent dans des objets issus du capital économique (e.g. dans des objets singuliers). La seconde section présente les cas de figure où ces significations à préserver s'ancrent dans le capital socioculturel (e.g. dans des pratiques). 


\section{Le capital économique en héritage}

Comme évoqué dans la partie sur les solidarités économiques, les chercheurs adoptent une vision centrée sur l'individu afin d'éclairer les choix de distribution de l'héritage économique. L'objectif étant d'identifier des variables de personnalité ou des motivations saillantes permettant de proposer aux entreprises et aux organisations liées à la construction/distribution de l'héritage des leviers d'actions pour se développer (Urien et Guiot, 2007). Ainsi, Malas (Malas, 2012; Malas et Guiot, 2010) montre que la manière dont les personnes âgées perçoivent l'avenir impacte directement leurs choix financiers tels l'épargne, les donations entre vifs ou encore les testaments patrimoniaux. Lorsque les individus n'ont pas d'enfant, les associations et autres institutions collectives peuvent prétendre être récipiendaires des dispositions testamentaires si elles arrivent à développer des valeurs proches de celles des potentiels donateurs (Krebs et al., 2012 ; Rieunier et Urien, 2011).

La majorité des travaux relatifs à la distribution du capital économique concerne la transmission d'objets possédant une forte valeur sentimentale. Des objets singuliers et différenciés qui sont qualifiés de «spéciaux » ou de possessions inaliénables car liées à des évènements de vie spécifiques et/ou à l'histoire collective d'une famille (Grayson et Shulman, 2000). Ainsi, les individus transfèrent des significations personnelles et familiales à des objets, et leurs objets à la génération suivante afin de préserver une partie de leur identité (tableau 4). Price et al. (2000) ont mis à jour une dualité entre ces logiques identitaires et des logiques relationnelles : les individus cherchent à identifier les destinataires les plus aptes à préserver les significations associées aux objets transmis. Très souvent, le choix du destinataire obéit à une règle de reproduction familiale : un objet qui se transmet de père en fils, de mère en fille ou à l'ainé.e de la famille par exemple (Price et al., 2000). De tels objets/pratiques produisent des hiérarchies sociales au sein des familles : certaines personnes endossent en effet le rôle de «gardien » de ces héritages - caretaker- et vont se sentir investies de la mission de les préserver et de les transmettre à leur tour (Curasi et al., 2004). Le destinataire n'est pas obligé d'accepter l'héritage mais, s'il le fait, les significations associées pourront servir de ressources mobilisables pour la construction de ses identités. Il convient de distinguer les significations associées à l'héritage (des souvenirs, une histoire, etc.) et les éléments affectifs et psychiques cristallisés par le processus de transmission. En effet, le rôle de destinataire peut être vécu de manière positive ou négative, comme un fardeau ou une obligation liée à un sentiment de dette (Curasi et al., 2004). Par exemple, Kessous et al. (2015) montrent que le don d'une montre de luxe d'un père à son fils entraîne ce dernier dans une logique de comparaison et engendre une série d'obligations (e.g. partager la passion du père, transmettre à son tour, être à la hauteur des attentes du père, etc.).

Tableau 4 : La transmission des possessions inaliénables

Pourquoi certaines possessions sont inaliénables?

Belk, 1988 ; Belk et al., 1988 ;

Belk, 2013, Grayson et Shulman, 2000 ; Richins, 1994
- Une possession inaliénable est une incarnation physique ou numérique (corporally indexical association) d'un lieu, d'une personne, d'une époque ou d'un évènement

- Les individus s'investissent personnellement pour entretenir la signification de ces objets

- La possession est valorisée pour sa signification indépendamment de sa valeur économique

- Le plus souvent la valeur n'est que symbolique. Lorsque la valeur est économique - propriété, entreprise familiale, etc.elle sera inaliénable si elle sert des objectifs bien définis 
dans le futur

Quels est le rôle d'une possession

inaliénable?

-Dans le cas d'une possession

valorisée par un individu

Belk, 1988 ; Curasi, 2011 ; Price et

al., 2000

-Dans le cas d'une possession valorisée par une famille

Curasi et al., 2004 ; McCracken, 1988

\section{Comment transmettre une} possession inaliénable?

Cours et al., 1999 ; Price et al., 2000 ; Stenvenson et Kates, 2010;

Wallendorf and Arnould, 1991 ;

Young et Wallendorf, 1989

\section{Pourquoi le statut inaliénable est} fragile lors du processus de transmission?

Bradford, 2009 ; Epp et Price, 2010 ; Kessous et al., 2015 ;

Lastovika and Fernandez 2005
- Elle permet à l'individu de renforcer et d'exprimer son identité, de se différencier des autres

- Elle sert de point d'ancrage et permet de garder un sens de contrôle sur le changement et sur le temps qui passe

- Elle permet de donner corps à l'identité et à l'histoire collective de la famille

- Elle produit une hiérarchie sociale dans la famille: une seule personne peut la détenir, mais elle ne lui appartient pas (il en est le gardien : le caretaker)

- Le transfert physique de l'objet se fait souvent lors des rites de passages traditionnels (communion, mariage, etc.) ou des rites de progression (anniversaires, etc.)

- Le transfert peut-être ritualisé de «caretaker» à «caretaker» ou plus spontané avec ou sans échange sur la signification

- Dans tous les cas, le détachement émotionnel n'est pas synchronisé au transfert physique

- L'objet va être transféré à un nouveau propriétaire qui va soit l'accepter soit le rejeter, mais en tout état de cause en transformer le sens

- Les objets peuvent retourner sur le marché et être désacralisés

\section{Le capital socioculturel comme support de significations à préserver}

Depuis Price et al. (2000), les chercheurs ont tenté d'identifier les autres moyens utilisés par les individus pour préserver des significations pour les autres générations. Ainsi, Guillemot (2015) montre que des récits de vie oraux ou écrits, des conversations et des moments passés ensemble sont des moyens privilégiés et valorisés pour faire passer des significations aux générations futures. Ces significations peuvent aussi s'incarner dans des pratiques de consommation porteuse d'une partie de l'identité familiale et collective. Ainsi, une «recette de grand-mère» (Kessous et Chalamon, 2014) ou des pratiques de consommation spécifiques peuvent se perpétuer de génération en génération et devenir des traditions familiales (Castano et al., 2010). On rejoint ici des problématiques partagées avec les recherches sur la socialisation du consommateur et particulièrement celles portant sur les traditions (Hirschman, 1985) et/ou sur le rôle de grands-parents (Derbaix et al., 2015 ; JosionPortail, 2014).

Si les pratiques de consommation actuelles peuvent être façonnées par les traditions issues du passé, elles peuvent également être envisagées comme des actes visant à préserver des significations et un monde viable pour les générations à venir. Ainsi, Urien et Kilbourne (2011) ont montré que les individus ayant un intérêt génératif prononcé - qui pensent que leurs actions ont un impact sur les générations futures - sont plus concernés par les comportements écoresponsables (recyclage, tri des déchets, etc.) que les individus ayant un faible intérêt génératif. Dans le prolongement de ces travaux, Lacroix et Jolibert (2015) ont élaboré une échelle de mesure qui permet d'identifier les consommateurs qui privilégient l'achat de produits et services qui préservent les conditions de vie (ou qui ne nuisent pas au 
bien-être) des générations futures. Guillemot et Urien (2016) expliquent ces transmissions entre générations démographiques en identifiant plusieurs «niveaux » ou «dimensions » au capital intergénérationnel. Les motivations à transmettre sont de nature «agentique » lorsqu'il s'agit de faire passer aux autres générations des significations personnelles issues de l'individualisation de son capital intergénérationnel. Les motivations peuvent être de nature «familiale » lorsque les significations sont liées à une identité, à un capital partagé par l'ensemble de la famille. Enfin, les motivations à transmettre peuvent également être de nature «communale » lorsqu'il s'agit de faire passer aux autres générations des significations collectives liées à un capital intergénérationnel partagé par l'ensemble d'une communauté ou d'une culture.

\section{DISCUSSION}

A l'issue de cette synthèse des connaissances en comportement du consommateur, nous sommes en mesure de proposer un cadre conceptuel intégrateur de la transmission intergénérationnelle (figure 1). Les sections suivantes vont montrer comment les trois éléments clés de ce cadre conceptuel vont permettre d'enrichir et de développer les travaux sur la transmission intergénérationnelle en comportement du consommateur. Les trois éléments clés sont les suivants. Premièrement l'articulation des préoccupations développementales des acteurs de la transmission autour du capital intergénérationnel. Le capital intergénérationnel étant défini comme un socle d'actifs matériels et immatériels qui se transmet et évolue de génération en génération. Deuxièmement, le cadre conceptuel précise la nature du capital intergénérationnel en distinguant les ressources socioculturelles, économiques, psychiques et génétiques. Enfin, il met en valeur les dimensions agentique, familiale et communale du capital intergénérationnel.

\section{L'articulation des préoccupations développementales des acteurs de la transmission}

Le premier apport du cadre conceptuel proposé est de relier les préoccupations développementales des destinateurs à celles des destinataires autour du capital intergénérationnel. Le destinateur va y transférer une partie de ses identités (personnelle, familiale et communale) en fonction de ses préoccupations développementales (apprendre, se distinguer, prendre soin, préserver). Le destinataire va y puiser des ressources pour construire ses propres identités et pratiques de consommation. Il va les adapter et les faire évoluer en fonction de son environnement et de ses propres préoccupations développementales. Traditionnellement les chercheurs en comportement du consommateur utilisent deux cadres d'analyse pour étudier les transmissions intergénérationnelles : celui de la socialisation fondé sur le rapport des jeunes consommateurs à leur capital socioculturel; et celui de la construction/distribution de l'héritage focalisé sur le rapport des personnes âgées à une partie de leur capital économique. Le cadre conceptuel intégrateur souligne qu'il existe des points de convergence entre la transmission d'objets intangibles et la transmission d'objets tangibles. En effet, les objets tangibles sont supports de significations et d'affects qui peuvent se transmettre sous des formes intangibles lors d'interactions continues (conversations, temps passés, pratiques de consommation, etc.). En outre, le cadre conceptuel intégrateur met en exergue deux autres préoccupations développementales en lien avec les phénomènes de transmission intergénérationnelle : se distinguer et prendre soi des autres générations.

Dans le premier cas (se distinguer des autres générations), les individus revisitent, réinterprètent et remodèlent leur capital intergénérationnel. L'état des connaissances a permis d'identifier quelques recherches en comportement du consommateur sur ce sujet (e.g. Ladwein et al., 2009 ; Moore et al., 2002). Celles-ci devraient se développer. En effet, les mutations numériques ou encore la nécessaire transition vers des modes de consommation 
durables vont rendre une partie des pratiques de consommation transmises par les parents inopérantes. Lorsque les modèles issus de la socialisation primaire (famille) et secondaire (école, pairs, médias) sont en accord, l'individu va reproduire et adapter son capital socioculturel. En revanche, une incompatibilité entre les deux modèles entraîne l'individu dans une période de remise en cause et de conversion, pouvant même aller jusqu'à l'échec de socialisation (Lahaye et al., 2007). Le défi consiste dès lors à comprendre pourquoi certaines pratiques sont plus ancrées que d'autres et à identifier les vecteurs facilitant le changement de pratiques de consommation. 
Figure 1. Cadre conceptuel intégrateur de la transmission intergénérationnelle en comportement du consommateur

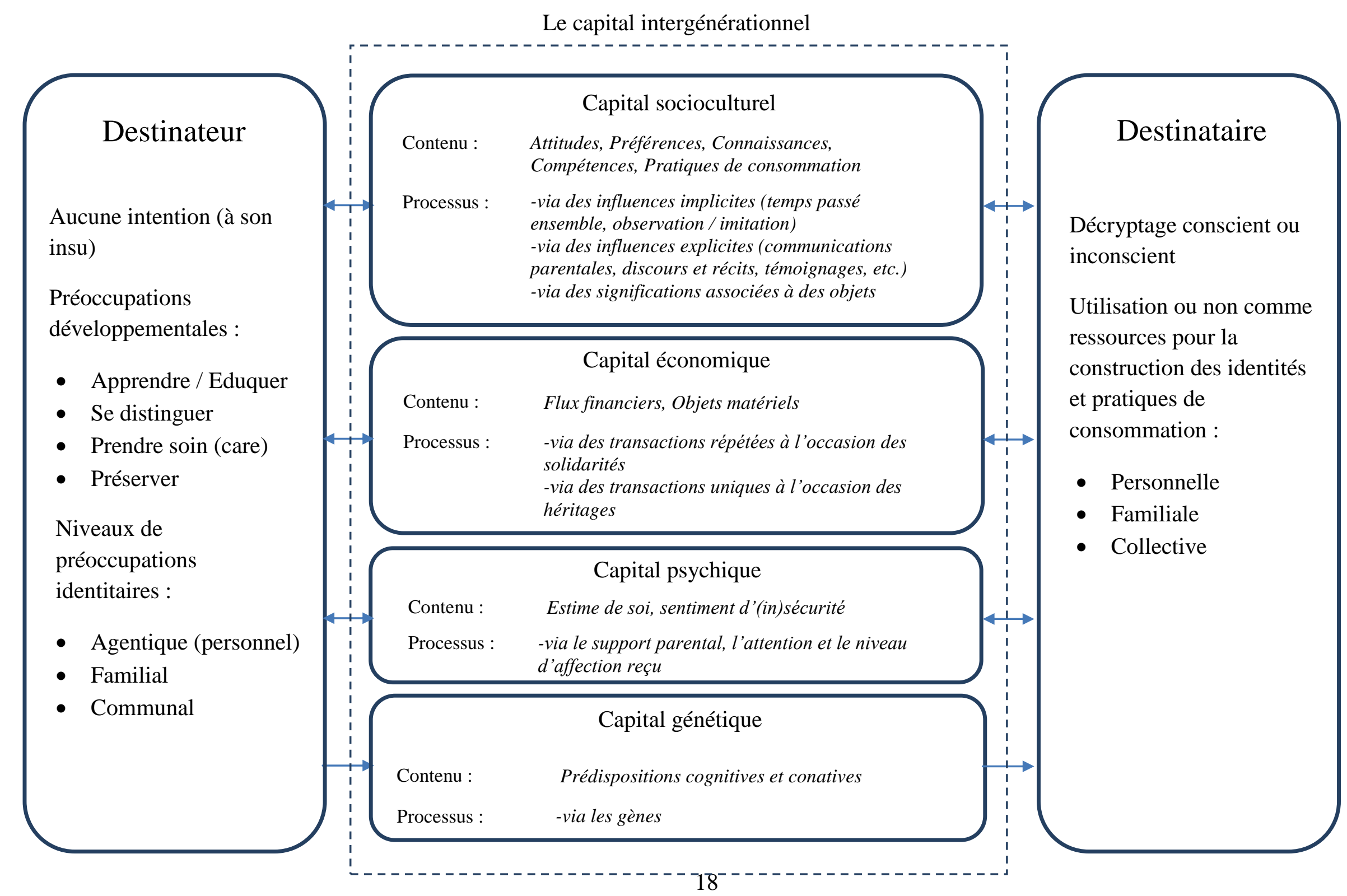


Dans le second cas, les individus cherchent à prendre soin (care) de leur capital intergénérationnel. Il existe peu de recherches en comportement du consommateur à ce sujet. Une clé d'entrée consiste à se focaliser sur le rôle de soutien (appelé respectivement caretaker dans les recherches concernant l'héritage et caregiver dans celles concernant les solidarités) de la génération pivot - celle des «30 et 65 ans ». Celle-ci est la grande oubliée des recherches sur la transmission intergénérationnelle. Elle se situe pourtant à un «carrefour générationnel » dans le sens où elle doit veiller au bien-être de ses enfants mais également à celui de ses parents qui deviennent progressivement dépendants (Olsen, 1999). Outre les solidarités financières (transfert d'argent, prêt d'un logement, don d'une voiture, etc.), les solidarités peuvent prendre la forme d'aides domestiques dans les tâches quotidiennes, mais aussi d'aides plus symboliques (mise en réseaux, échange de connaissance, temps passé, écoute, présence, etc.) (Attias-Donfut, 2000 ; Masson, 2009). Les recherches devront vérifier les hypothèses de déterminisme familial (le genre, le rang dans la fratrie, etc.), s'intéresser aux émotions ressenties et aux conséquences de ces solidarités sur les pratiques de consommation. A quoi renonce-t-on? Que change-t-on ? Quels services développer pour soutenir «l'axe de stabilité de la famille » (Attias-Donfut, 2000) ? Toutes les conditions sont réunies pour que les recherches sur la thématique se développent car elles répondent à des préoccupations économiques (l'augmentation de la précarité) et sociétales (le vieillissement démographique) majeures.

\section{La nature du capital intergénérationnel}

Le second apport du cadre conceptuel proposé est de préciser la nature du capital intergénérationnel. Il distingue les ressources socioculturelles, économiques, psychiques et génétiques qui traversent les générations. Précisons qu'il ne faut pas voir ces catégories comme exclusives car, en réalité, le capital intergénérationnel est constitué de couches qui interagissent et se superposent les unes aux autres : Le capital socioculturel (compétences, connaissances et attitudes en matière de consommation) traverse les générations grâce à une combinaison d'influences implicites (processus d'observation/imitation) et explicites (pratiques éducatives). Le passage entre les générations est interactif : les plus âgés apprennent aux plus jeunes qui en retour actualisent les pratiques et influencent les plus âgés. Le capital économique, quant à lui, traverse les générations sous la forme de flux financiers et d'objets matériels à l'occasion des solidarités et des héritages. Une partie des ressources économiques - notamment les possessions inaliénables - est porteuse de significations qui enrichissent le capital socioculturel. Le capital psychique est constitué des ressources psychiques sur lesquelles peuvent ou non s'appuyer les individus. Ces ressources psychiques se transmettent à travers le soutien et l'attention parentale et s'incarnent dans le niveau d'estime de soi ou le sentiment d'in/sécurité perçue. Les recherches récentes sur la socialisation du consommateur laissent à penser que ces ressources peuvent impacter le développement psychologique de l'individu qui va, à son tour, influencer la manière dont il va intégrer et s'approprier des valeurs, objets et pratiques de consommation. Enfin, le capital génétique concerne les prédispositions génétiques à effectuer/s'engager dans des choix/comportements. Il a un statut particulier puisque le transfert ne se fait que dans un seul sens.

Le cadre conceptuel proposé ici suggère d'enrichir la compréhension des phénomènes de transmission intergénérationnelle en prenant en compte les effets d'interactions entre ces différentes ressources héritées. Si la nature du capital socioculturel est bien documentée en comportement du consommateur, ce n'est pas le cas des ressources économiques et encore 
moins des ressources psychiques et génétiques. Les trois paragraphes suivants donnent des pistes pour les prendre en compte.

En ce qui concerne le capital économique, les recherches se focalisent essentiellement sur la transmission des objets à valeur sentimentale. Les flux financiers et les conditions de vie matérielles sont peu étudiés. Pourtant, ils déterminent et influencent fortement les conditions de vie et les choix familiaux et personnels de ceux qui le reçoivent (Piketty, 2013). Il conviendrait d'approfondir la compréhension de ces mécanismes d'influence. On pourra étudier les effets directs du capital économique sur les pratiques de consommation, ou indirectement via le développement de valeur spécifique. Par exemple Lahaye et al. (2007) montrent que des personnes évoluant dans un environnement économique défavorable développent des caractéristiques de personnalité spécifiques (démarche critique, réflexive et créatrice peu développée). On peut dès lors se demander si la reproduction intergénérationnelle des pratiques de consommation ne serait pas plus fréquente lorsque le capital économique est peu développé.

Le concept d'attachement (Bowbly, 1978) utilisé en psychologie et plus particulièrement dans les travaux en psychiatrie et psychologie clinique permettrait d'enrichir la réflexion et de mieux comprendre le rôle des ressources psychiques. En effet, selon Bowbly, les liens affectifs tissés dans l'enfance ont une influence déterminante sur la manière dont l'enfant va percevoir son environnement à travers la confiance qu'il place en lui, son niveau d'empathie ou encore d'estime qu'il a de lui-même. Ainsi si le besoin d'attachement est satisfait, l'individu pourra développer plus facilement des comportements de générosité, de partage ou d'empathie - comme le don par exemple. En revanche, un besoin d'attachement non satisfait entraine l'individu dans l'adoption de comportements non valorisés socialement (comportement compulsif, adoption de valeurs non désirables). Ainsi, la transmission intergénérationnelle ne s'accompagne pas toujours d'effets positifs mais peut, au contraire, véhiculer des comportements négatifs. De manière opérationnelle, on pourrait alors vérifier l'influence des ressources psychiques sur des comportements non désirables de manière indirecte à travers les effets de la confiance en soi, du niveau d'empathie, ou encore de l'estime de soi.

Enfin, les méthodologies fondées sur la comparaison de jumeaux - vrai/faux jumeaux élevés ensemble et/ou séparément - permettent de clarifier la nature des interactions entre le capital génétique et les conditions environnementales du développement de l'individu (Johnson et al., 2009). Concrètement, il s'agit de soumettre une fratrie à des questionnaires et de comparer les réponses en isolant les effets imputés au capital génétique, de ceux inhérents aux ressources socioculturelles et économiques (liées à l'environnement partagé qui fait référence aux conditions de vie et d'éducation) et aux ressources psychiques (à partir de l'environnement non partagé qui fait référence aux expériences personnelles et aux ressentis des membres de la fratrie). Ces méthodes offrent des résultats satisfaisants en biologie comportementale même si elles sont difficiles à mettre en œuvre et souffrent de biais de validité liés à la taille des échantillons. Il s'agit donc d'un défi de taille mais comme le souligne Harari (2015 :277) : «Les scientifiques sont de plus en plus enclins à soutenir que le comportement humain est déterminé par les hormones, les gènes et les synapses [...] pendant combien de temps encore pourrons-nous maintenir le mur qui sépare le département de biologie des facultés de sciences humaines?».

\section{Les dimensions du capital intergénérationnel}

Dans le prolongement, le troisième apport de ce cadre est de mettre en valeur plusieurs niveaux de préoccupations identitaires lors des transmissions intergénérationnelles : agentique 
(lié à l'identité personnelle), familial (lié à l'identité familiale) et communal (lié à l'identité collective d'une communauté). Autrement dit, il existe plusieurs dimensions au capital intergénérationnel dont certaines sont individualisées, d'autres partagées avec l'ensemble de la famille, et d'autres encore communes à la communauté d'appartenance. La dimension communale de la transmission intergénérationnelle permet d'appréhender comment des éléments du capital intergénérationnel peuvent se transmettre entre générations qui ne se côtoient pas directement (les générations démographiques).

Il s'agit maintenant de préciser et d'éclairer les niveaux agentique, familial et communal du capital intergénérationnel. S'expriment-ils de la même façon lorsqu'il s'agit d'éducation, de solidarité et de préservation? L'élaboration d'outils de mesure fiables et valides permettrait de répondre à ces questions. Actuellement, les échelles sont utilisées du côté des recherches sur l'héritage (Guillemot et Urien, 2016; Lacroix et Jolibert, 2015 ; Urien et Kilbourne, 2011). Néanmoins, celles-ci s'ancrent dans une vision de la générativité qui s'appuie principalement sur le désir de préservation (McAdams et de StAubin, 1992). Un retour à la théorie originelle d'Erikson (1959) permettrait de prendre en compte les préoccupations liées à l'éducation et au soin.

\section{CONCLUSION}

L'objectif de l'article est de proposer un cadre conceptuel de la transmission intergénérationnelle intégrant l'ensemble des connaissances en comportement du consommateur sur le sujet. Une lecture eriksonienne de la transmission intergénérationnelle permet de mettre en évidence quatre positions des individus vis-à-vis de leur capital intergénérationnel : s'en imprégner, s'en distinguer, en prendre soin et le préserver. Un état des connaissances organisé autour de ces quatre positions précise la nature du capital intergénérationnel en distinguant les ressources socioculturelles, économiques, psychiques et génétiques. De la même manière il a mis en évidence des dimensions individuelle, familiale et communale au capital intergénérationnel.

Ce cadre conceptuel offre ainsi une vision plus complète des transmissions intergénérationnelles. Il ouvre également des perspectives de recherche. Parmi celles exposées dans la partie discussion retenons celles-ci : étudier les relations d'interactions entre les différentes composantes du capital intergénérationnel en prenant en compte les conditions de vie matérielles, des variables psychologiques telles l'estime de soi ou la confiance en soi, et/ou en développant des méthodologies basées sur la comparaison des fratries. Il convient également de développer des connaissances permettant d'accompagner les individus dans l'individualisation de leur capital socioculturel dans un contexte de bouleversement des pratiques de consommation lié aux transitions numériques et énergétiques. Ou encore de mieux comprendre les difficultés affrontées par la génération «pivot» lorsqu'elle doit prendre soin de son capital intergénérationnel. Notons enfin la nécessité de développer des instruments permettant de mesurer les différentes préoccupations développementales liées à la transmission intergénérationnelle.

Ces connaissances nouvelles pourront désormais être développées dans un cadre commun. La dispersion des connaissances sous des mots-clés diversifiés souligne une limite de cette recherche. En effet seuls les travaux dont les mots-clés sont « socialisation », « influences », « apprentissages » « héritage », «échanges », « dons » ou encore «legs » ont été considérés. Cela peut expliquer l'absence de certains travaux dans l'état des connaissances présentée. Par exemple, une partie des recherches sur la consommation ethnique auraient pu y figurer dans la mesure où un bon nombre d'entre elles considèrent une dimension «héritage » (ÖzçăglarToulouse et al., 2009). D'autres travaux en marketing du tourisme/culturel montrent que des 
sites/musées sont valorisés pour leur capacité à préserver et à mettre en valeur l'héritage collectif et l'histoire commune (Bourgeon-Renault et al., 2009; Leong et al., 2015). L'ensemble de ces travaux pourrait enrichir la compréhension de la dimension communale du capital intergénérationnel qui permet d'expliquer les transmissions entre les générations démographiques.

Dans tous les cas, nous espérons que ce cadre conceptuel permettra aux chercheurs de mieux appréhender les phénomènes de transmission intergénérationnelle et d'y développer de nouvelles connaissances. Il s'agit d'un sujet majeur avec des impacts sociétaux et qui répond à des défis de la société de demain : éduquer les jeunes consommateurs, les accompagner vers l'adoption de pratiques de consommation durables, ou encore prendre soin et préserver les aspects du capital intergénérationnel dans un contexte de précarité économique et de vieillissement démographique.

\section{BIBLIOGRAPHIE}

Arndt J (1971) A research Note on Intergenerational Overlap of Selected Variables. Markeds Kommunikasjon 3: 1-8

Attias-Donfut C (2000) Rapports de générations. Transferts intrafamiliaux et dynamique macrosociale. Revue française de sociologie 41(4) : 643-684

Attias-Donfut C et Segalen M (1998) Grands-parents, La famille à travers les générations. Paris : Editions Odile Jacob

Arrondel L et Masson A (2011) Taxer les héritages pour accroître la mobilité du patrimoine entre générations. Revue française d'économie. XXVI(2) : 23-72

Axim W.G, Pearce L.D et Ghimire D. (1999) Innovations in life history calendar applications. Social Science Research 28: 243-264

Ayadi K et Gollety M (2012) La famille : un lieu d'échanges privilégiés pour apprendre à consommer. In : Brée J. (ed) Kids Marketing, éditions EMS, $2^{\text {nd }}$ edition, pp149-176

Beatty SE et Talpade S (1994) Adolescent Influence in Family Decision Making: A replication with Extension. Journal of Consumer Research 21 (September): 332-41

Belk RW (1988) Possessions and the Extended Self, Journal of Consumer Research 15(2): 139-168

Belk RW (2013) Extended Self in a Digital World. Journal of Consumer Research 40 (3): 477-500

Belk RW, Sherry Jr. J-F et Wallendorf M (1988) The sacred and the Profane in Consumer Behavior: Theodicy on the Odyssey. Journal of Consumer Research 16 (June): 1-38

Bourcier-Béquaert B et de Barnier V (2010) Pour un cadre élargi du concept de génération en marketing. Recherche et Applications en Marketing 25 (3) : 115-135

Bourdieu P (1979) La distinction : critique sociale du jugement. Paris : Les Editions de Minuit

Bourdieu P (1994) Raisons Pratiques sur la théorie de l'action. Paris : Editions du Seuil

Bourgeon-Renault D, Urbain C, Gombault A, Le Gall-Ely M et Petr C. (2009) Gratuité et valeur attachée à l'objet de consommation culturelle : le cas des musées et des monuments Décisions Marketing, 54 (Avril-Juin) : 51-62

Bowbly J (1978) Attachement et perte, vol. 1 : L'attachement. Paris : PUF.

Bradford TW (2009) Intergenerationally Gifted Asset Dispositions. Journal of Consumer Research 36(1): 93-111

Butler RN (1963) The Life Review: an interpretation of reminiscence in the age. Psychiatry 26: $65-75$ 
Cai Y, Zhao G et He J (2015) Influences of two modes of intergenerational communication on brand equity. Journal of Business Research 68 (3): 553-560

Carlson L et Grossbart S (1988) Parental style and consumer socialization of children. Journal of Consumer Research 15 (1): 77-94

Carlson L, Walsh A, Laczniak R et Grossbart S (1994) Family communication patterns and marketplace motivations, attitudes, and behaviors of children and mothers. Journal of Consumer Affairs 28: 25-53

Castano R, Perez ME et Quintanilla C (2010) Cross-border shopping: family narratives. Qualitative Market Research 13 (1): 45-57

Chaplin LN et Roedder-John D (2010) Interpersonal influences on adolescent materialism: A new look at the role of parents and peers. Journal of Consumer Psychology 20: 176-184

Childers TL et Rao AR (1992) The Influence of Familial and Peer-based Reference Groups on Consumer Decisions. Journal of Consumer Research 19 (2) : 198-211

Cordier M, Houdré C et Ruiz H (2007) Transferts intergénérationnels entre vifs : aides et donations. INSEE Première : $\mathrm{n}^{\circ} 1127$

Cotte J et Wood SL (2004) Families and Innovative Consumer Behavior: A Triadic Analysis of Sibling and Parental Influence. Journal of Consumer Research 31 (1): 78-86

Cours D, Heisley DD, Wallendrorf M et Johson D (1999) It's all in the family, but I want it. In Arnould E et Scott L (eds) Advances in Consumer Research. UT: Association for Consumer Research 26: 253-29

Curasi CF (2011) Intergenerational possession transfers and identity maintenance. Journal of Consumer Behaviour 10 (2): 111-118

Curasi CF, Price LL et Arnould EJ (2004) How Individuals' Cherished Possessions Become Families' Inalienable Wealth. Journal of Consumer Research 31(3) : 609-622

Decoopman I et Gentina E (2013) La propension des mères à échanger des vêtements avec leur fille adolescente : quelle conséquences pour le marketing ? Décisions Marketing 70 : 75-90

Derbaix M, Derbaix C, Kindt M, Korchia M et Luna Fernandez M. (2016) Transmissions culturelles entre parents et enfants : le cas des goûts musicaux. Management \& Avenir 87 (5) : 151-174

Derbaix C, Leheut E, Kindt M et Boi L (2015) Les grands-parents : Agents d'une socialisation spécifique de leurs petits-enfants consommateurs? Revue Française du Marketing 252: 7-22

Downey H et Ellis S (2008) Tails of animal attraction: Incorporating the feline into the family. Journal of Business Research 61(5): 434-441

Epp AM et Price LL (2010) The Storied Life of Singularized Objects: Forces of Agency and Network Transformation. Journal of Consumer Research 36 (5): 820-837

Erikson E (1950) Childhood and Society. W.W. Norton \& Co

Erikson E (1959) Identity and the life cycle: Selected paper. Psychological Issues 1 : 50-100

Felfoul KS (2014) Le rôle de la famille dans le développement du matérialisme : une étude basée sur le paradigme du parcours de vie. Thèse de Doctorat soutenue à l'Université de Laval, Québec

Gale WG et Schultz JK (1994) Intergenerational transfers and the accumulation of wealth. Journal of Economic perspective 38: 145-160

Gentina E, Decoopman I et Ruvio A (2013) Social comparison motivation of mother's with their adolescent daughters and its effects on the mother's consumption behavior. Journal of Retailing and Consumer Services 20: 94-101

Gire P (2003) Epistémologie du concept de transmission. In Rodet C (Ed) La transmission dans la famille : secrets, fictions et idéaux. L'Harmattan, pp 19-24 
Gollety M (1999) Lorsque parents et enfants s'apprennent mutuellement à consommer... Décisions Marketing 18 : 69-80

Gollety M, Guichard N et Ayadi K (2012) La consommation dans la famille : des influences de nature différente. In Brée J (ed) Kids Marketing. éditions EMS, $2^{\text {nd }}$ édition, pp 223-254

Grayson K et Shulman D (2000) Indexical and the verification function of irreplaceable possessions: A semiotic analysis. Journal of Consumer Research 27 (1): 17-30

Guillemot S (2015) La transmission intergénérationnelle: regard sur les services aux particuliers. Revue Management \& Avenir 77 (3) :51-69

Guillemot S et Urien B (2010) La rédaction d'une histoire de vie chez les personnes âgées : fondements conceptuels, dimensionnement et proposition d'une échelle de mesure des motivations au récit de vie. Recherche et Applications en Marketing 25 (4) : 25-43

Guillemot S et Urien B (2016) Legacy Writing and the consumption of biographic services. Psychology \& Marketing 33 (11): 971-981

Harari YN (2015) Sapiens, une brève histoire de l'humanité. Albin Michel

Heckler SE, Childers TL et Arunachalam R (1989) Intergenerational influences in adult buying behaviors: an examination of moderating factors. In Srull TK (Ed.) Advances in Consumer Research, 16, Provo, UT, Association for Consumer Research, pp 276-284

Hémar-Nicolas V et Rodhain A (2012) Pour un Kids Marketing responsables !!! In Brée J. (Ed) Kids Marketing. éditions EMS, $2^{\text {nd }}$ edition, pp 71-116

Hirschman EC (1985) Primitive Aspects of Consumption in Modern American Society. Journal of Consumer Research 12(2): 142-154

Johnson W, Turkheimer E, Gottesman I et Bouchard T (2009) Beyond Heritability: Twin Studies in Behavioral Research. Current Directions in Psychological Science 18 (4): 217220

Josion-Portail M (2014) Comment parents et grands-parents influencent l'apprentissage du bien manger chez l'enfant: Contribution de la transmission intergénérationnelle dans le contexte de la consommation alimentaire. Thèse de doctorat en Sciences de Gestion soutenue à l'Université de Paris 1

Josion-Portail M et Michel G (2016) Comment favoriser la transmission intergénérationnelle du "bien manger" ? Vers de nouveaux leviers au service des pouvoirs publics, Actes du $32^{\text {ème }}$ congrès de l'Association Française de Marketing, Lyon

Karanika K et Hogg M (2016) Consumption through the Ambivalent Prism of Intergenerational Support. European Journal of Marketing 50 (3/4): 575-601

Kaës R, Faimberg H, Enriquez, Baranes J-J (2003) Transmission de la vie psychique entre générations. Dunod, $2^{\text {nd }}$ édition

Kessous A, de Barnier V et Valette-Florence P (2015) «A la recherche du temps perdu » : la transmission d'objets de luxe de père en fils, entre cadeau et fardeau. Décisions Marketing $80: 17-34$

Kessous A et Chalamon I (2014) La transmission de remèdes de grand-mères de mère en fille : une extension temporelle de soi. Décisions Marketing $76: 61-73$

Kim C, Lee H et Tomiuk MA (2009) Adolescents' perceptions of family communication patterns and some aspects of their consumer socialization. Psychology \& Marketing 26 (10) : 888-907

Krebs A, Rieunier S et Urien B (2012) Le legs aux organismes culturels : identification du testateur et pratiques managériales. In Guiot D. et Urien B. (eds) Comprendre le consommateur âgé, nouveaux enjeux et perspectives. Bruxelles : De Boeck: 157-178

Lacroix C et Jolibert A (2015) Targeting consumers who care about future generations. Psychology \& Marketing 32 (8): 783-794 
Ladwein R, Carton A et Sevin E (2009) Le capital transgénérationnel : la transmission des pratiques de consommation de la mère vers sa fille. Recherche et Applications en Marketing 24 (2) :1-27

Lahaye W, Poutois J-P et Desmet H (2007) Transmettre d'une génération à l'autre. Paris : PUF

Lastovicka JL et Fernendez KV (2005) Three Paths to disposition: The movement of Meaningful possessions to strangers. Journal of Consumuer Research 31 (march): 813-23

Lee E, Mathur A, Kwai Fatt C et Moschis G.P (2013) The Timing and Context of Consumer Decisions: Insights from the Life Course Paradigm. Marketing Letters 23(3): 793-805

Leong AW, Yeh S, Hsiao Y et Huan TT (2015) Nostalgia as travel motivation and its impact on tourists' loyalty. Journal of Business Research 68 (1): 81-86

Lueg JE, Ponder N, Beatty SE et Capella ML (2006) Teenagers' use of alternative shopping channels: A consumer socialization perspective. Journal Of Retailing 82 (2):137-153

Mahur H et Moschis GP (1999) Exploring the Intergenerational Caregiver Market: A Study of Family Care Providers for the Elderly. Journal of Marketing Theory and Practice, 7 (3):76-86

Malas Z et Guiot D (2010) Une mesure de la Valence de la perspective / Future (VPF) des personnes âgées : conséquences sur les choix financiers. Revue Sciences de Gestion 76 : $65-91$

Malas Z (2012) Le rapport à l'avenir du consommateur âgé : entre épargne et consommation. In Guiot D et Urien B (eds) Le consommateur âgé. Bruxelles : De Boeck, pp :51-66

Mandrik CA, Fern EF, et Bao Y (2005) Intergenerational influence: Roles of conformity to peers and communication effectiveness. Psychology \& Marketing 22 (10) : 813-832

Masson A (2009) Des liens et des transferts entre générations. Paris : Editions EHESS

McAdams DP (2001) Generativity in midlife. In Lachman (Ed), Handbook of midlife development. New York : Wiley, pp 395-443

McAdams DP et de St. Aubin E (1992) A theory of generativity and its assessment through self-report, behavioral acts, and narrative themes in autobiography. Journal of personality and social psychology 62:1003-1015

McCracken G (1988) Lois Roget: Curatorial Consumer in a Modern World. In McCraken G (ed) Culture and Consumption. Bloomington: Indiana University Press, pp: 44-56

Moore-Shay ES et Lutz RJ (1988) Intergenerational Influences in the Formation of Consumer Attitudes and Beliefs About the Marketplace: Mothers and Daughters. Advances in Consumer Research 15 (11): 461-467

Moore ES, Wilkie WL et Lutz RJ (2002) Passing the Torch: Intergenerational Influences as a Source of Brand Equity. Journal of Marketing 66 (2): 17-37

Moschis GP et Churchill Jr. GA (1978) Consumer Socialization: A Theoretical and Empirical Analysis. Journal of Marketing Research 15 (4): 599-609

Moschis GP et Moore RL (1979) Family communication and consumer socialization. Advances in Consumer Research 6 (1): 359-363

Moschis GP, Moore RL et Smith RB (1984) The impact of family communication on adolescent consumer socialization. Advances in Consumer Research 11 (1): 314-319

Nancarrow C, Tinson J. et Brace I. (2008) Consumer savvy and intergenerational effects. International Journal of Market Research, 50 (6): 731-755

Noble SM, Schewe CD et Kuhr M (2004) Preferences in health care service and treatment: A generational perspective. Journal of Business Research 57 (9): 1033-1041

Obermiller C et Spangenberg ER (2000) On the Origin and Distinctness of Skepticism toward Advertising. Marketing Letters 11 (4): 311-322 
Olsen B (1999) Exploring Women's Brand Relationships and Enduring Themes At Mid-Life. In Arnould E et Scott LM (Eds) Advances in Consumer Research. Provo, UT : Association for Consumer Research, 26: 615-620

Özçăglar-Toulouse N, Béji-Bécheur A, Fosse-Gomez M., Herbert M. et Zouaghi S. (2009) L'ethnicité dans l'étude du consommateur : un état des recherches. Recherche et Applications en Marketing 24(4) : 57-76

Piketty T (2013) Le capital au XXI ${ }^{e}$ siècle. Paris : Editions du Seuil

Plomin R, DeFries J, McClean G et Rutter M (1999) Des gènes au comportement, introduction à la génétique comportementale. DeBoeck Université, 3ème édition

Préel B (2005) Les générations mutantes : Belle époque, krach, mai 68, Internet, quatre générations dans l'histoire. Paris : La Découverte

Price LL, Arnould EJ et Curasi CF (2000) Older Consumers' Disposition of Special Possessions. Journal of Consumer Research, 27 (2): 179-201

Richins ML (1994) Valuing Things: The public and private meaning of Possessions. Journal of Consumer Research 21 (December): 504-521

Richins ML et Chaplin LN (2015) Material Parenting: How the Use of Goods in Parenting Fosters Materialism in the Next Generation. Journal of Consumer Research 41:1333-1357

Rieunier S et Urien B (2011) Léguer, c'est continuer à vivre. In Bergadaà M, Le Gall-Ely M et Urien B (Eds) Don et pratiques caritatives. Bruxelles : De Boeck University

Rindfleisch A, Burroughs JE et Denton F (1997) Family Structure, Materialism, and Compulsive Consumption. Journal of Consumer Research 23 (4): 312-325

Rindfleisch A, Burroughs JE et Wong N (2009) The Safety of Objects: Materialism, Existential Insecurity, and Brand Connection. Journal of Consumer Research 36 (1): 1-16.

Roberts JA, Manolis C et Tanner Jr JF (2003) Family Structure, Materialism and Compulsive Buying: A Reinquiry and Extension. Academy of Marketing Science 31 (3): 300-311

Roedder John D (1999) Consumer Socialization of Children: A retrospective Look of Twenty-Five years of Research. Journal of Consumer Research 26 (December): 183-213

Schindler RM, Lala V et Corcoran C. (2014) Intergenerational Influence in Consumer Deal Proneness. Psychology \& Marketing, 31 (5): 307-320

Shah RH et Mittal B (1997) Toward a Theory of Intergenerational Influence in Consumer Behavior: An Exploratory Essay. Advances In Consumer Research, 24 (1): 55-60

Siddiqui K, Bashir S, Sarki IH, Jaffari SIA et Abbas S (2012) Intergenerational Influences on Brand Preferences: Consumables Vs Durables. International Journal of Business and Social Science 13 (3): 227-231

Simonson I, et Sela A (2011) On the Heritability of Consumer Decision Making: An Exploratory Approach for Studying Genetic Effects on Judgment and Choice. Journal of Consumer Research 37(6): 951-966

Stevenson GJ et Kates SM (1999) The last gift: the meanings of gift-giving in the context of dying of AIDS. In Arnould EJ et Scott L (Eds) Advances in Consumer Research, Association for Consumer Research, Provo, UT, Vol. 26, pp 113-18

Tavoillot P-H, Attias-Donfut C, Masson A, Van de Velle C et Guérin S. (2011) Métarevue sur le lien intergénérationnel : Etat des savoirs et Etat des débats. Campus Lab MACIF

Trizzulla C, Garcia-Bardidia R et Rémy E (2016) De Bourdieu à Lahire : déterminismes sociaux et pratiques de consommation plurielles. Recherche et Applications en Marketing 31 (1): 87-105

Turkheimer E (2000) Three Laws of Behavior Genetics and What They Mean. Current Directions in Psychological Science 9 (5): 160-64

Urien B (2003) L'anxiété face à la mort et son influence sur le comportement du consommateur. Recherche et Applications en Marketing, 18(4) : 23-41 
Urien B et Guiot D (2007) Attitude face à la mort et comportement d'ajustement des consommateurs âgés: vers l'élaboration d'une réponse marketing. Décisions Marketing 46 (avril-juin) : 23-36

Urien B et Kilbourne W (2011) Generativity and self-enhancement values in eco-friendly behavioral intentions and environmentally responsible consumption behavior. Psychology \& Marketing 28 (1): 69-90

Vaillant GE (2002) Aging well. Boston: Little Brown

Viswanathan M, Childers TL, et Moore ES (2000) The Measurement of Intergenerational Communication and Influence on Consumption: Development, Validation, and CrossCultural Comparison of the IGEN Scale. Journal of the Academy of Marketing Science 28 (3): 406-424

Wallendorf M et Arnould EJ (1991) "We" Gather Toegether': Consumption rituals of thanksgiving day. Journal of Consumer Research 18 (June): 13-31

Ward S (1974) Consumer Socialization. Journal of Consumer Research 1 (September): 1-14

Ward S, Wackman D et Wartella E (1977) The Development of Consumer InformationProcessing Skills: Integrating Cognitive Development and Family Interaction Theories. In Perreault WD Jr (ed) Advances in Consumer Research vol 4. Atlanta, GA : Association for Consumer Research, Pages: 166-171

Wiman AR (1983) Parental influence and children's response to television advertising. Journal of Advertising 12 (1): 12-18

Winter J-P (2012) Transmettre (ou pas). Albin Michel

Woodson LG, Childers T et Winn PR (1976) Intergenerational Influences in the purchase of Auto Insurance. In Locander W (Ed) Marketing Looking Outward. American Marketing Association : Business proceeding 38: 43-49

Young M et Wallendorf M (1989) Ashes to Ashes, Dust to Dust: Conceptualizing Consumer Disposition of possessions. American Marketing Association Winter Educators' Conference Proceedings. Chicago: American Marketing Association, pp :33-39 